\title{
Adapted Design of Process Multi-Stage Centrifugal Compressor and Comparison with Available Data
}

\author{
H. Ali Marefat, M. R. Shahhosseini, and M. A. Ashjari
}

\begin{abstract}
Process multi-stage centrifugal compressors have a very wide range of applications in oil, gas and petrochemical industries. One of the major applications is in gas pipeline transports. In this paper, design procedure of process multi-stage centrifugal compressor, by considering one dimensional flow design, is presented. By taking into account that the procedure is specially targeted to design a compressor for gas industry and considering that the ability for test of these kinds of compressors is already available at OTCE Company, therefore result achieved by mentioned procedure is compared by empirical data. In addition, aerodynamics and thermodynamics gas flow governing equations are derived and appropriate correction factors are employed in every individual section in order to fulfill complete design procedure. In this procedure, working fluid flow is assumed as one dimensional flow and considered as real gas. Moreover, the procedure is started from calculation of impeller inlet and it is continued for the other sections including impeller exit, diffuser and volute. Besides, total efficiency, stage efficiency, correction factors and leakages are calculated along with other sections calculations. Finally, the obtained design results are compared with experimental data.
\end{abstract}

Index Terms - Centrifugal compressor, multi-stage, design.

\section{INTRODUCTION}

Multistage centrifugal compressors (Fig. 1) play essential role in oil, gas, refinery, refrigerating, air conditioning, etc. industries. Due to their wide range of application, these kinds of compressors are so interesting for researchers and engineers to work on. For the first time, late in $19^{\text {th }}$ century centrifugal compressor was invented by Auguste Rateau. By 1899 he had a single-stage prototype on his test stand compressing $0.5 \mathrm{~m}^{3} / \mathrm{s}$ of atmospheric air to a discharge pressure of 1.5 bar (absolute) at a rotational speed of 20,000 rpm [1].

In this paper, by considering all mentioned necessities, adapted design procedure of multistage centrifugal compressor under real gas circumstances as well as one dimensional flow is presented. The procedure is started by preliminary design calculation for the whole compressor. Preliminary calculation results are fed to other sections calculations as initial values and by employing numerical iteration methods, key parameters for every individual

Manuscript received January 4, 2013; revised February 20, 2013

H. A. Marefat is with Islamic Azad University, Science \& Research Campus, Tehran, Iran (email: halimarefat@gmail.com).

M. R. Shahhosseini is with Oil TurboCompressor Equipment Co. (OTCE). Ph.D., Manager of Design Department (e-mail: shahhosseini@cgc-co.com).

M. A. Ashjari is with the Mechanical Engineering Department, Islamic Azad University, Jolfa branch, Assistance Professor (e-mail: maliashjari@iauj.ac.ir). section are achieved. Besides, total efficiency, stage efficiency, correction factors and leakages are calculated along with the procedure. Finally, obtained design results are compared with empirical data.

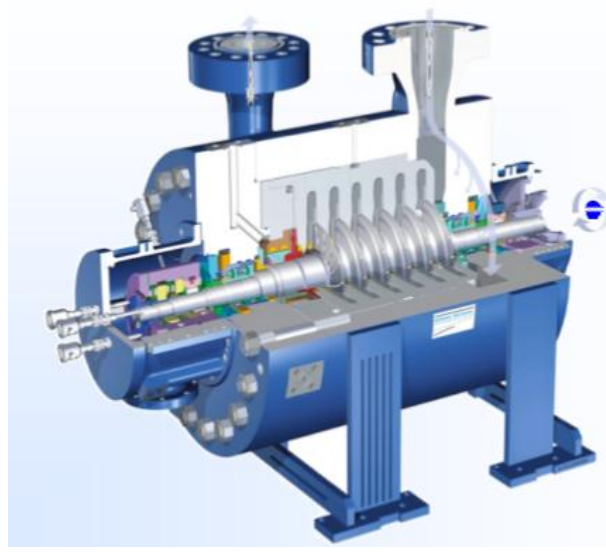

Fig. 1. Multistage centrifugal compressor.

\section{PRELIMINARY DESIGN}

In the very first step of procedure, there are just a few known parameters. Therefore, approximate values for vital parameters should be determined, which will be corrected further by suitable coefficients in the future steps. In this step, suction pressure, discharge pressure, suction temperature and mass flow are given in order to obtain numerical result for rough compressor sizing. Moreover, the procedure must be provided with gas components which are needed to use in an appropriate equation of state so as to determine pseudo-critical properties of working fluid. In this paper based on gas chemical mixture and reasons mentioned in [1] and [2], LKP equation of state is employed. The equation of state makes available compressibility factor, volume isentropic exponent and temperature isentropic exponent, which help to calculate process suction volume flow from eq. 1.

$$
\dot{V}_{o t}=\frac{\dot{m} Z_{0 t} R T_{0 t}}{p_{0 t}}
$$

Furthermore, it is important to know how many stages would be contained in a casing. To specify number of stages, compressor total polytropic head is calculated by eq. 2 .

$$
y_{p}=T_{s} Z R \frac{\kappa \eta_{p}}{\kappa-1}\left[\pi^{\frac{\kappa-1}{\kappa \eta_{p}}}-1\right]
$$

where $\pi, \kappa$ and $Z$ are pressure ratio, average isentropic exponent and average compressibility factor respectively. Now, number of stages can be obtained from the following correlation: 


$$
\text { Number of stage }=\frac{y_{p}}{y_{p \max }}+1
$$

Dividing total polytropic head by number of stages gives the exact value for stage polytropic head, from which discharge pressure of each stage can be calculated.

To make sure that the compressor is rotordynamically safe, tip speed should be obtained in order to feed in Eq. 4 to determine tip speed Mach number, which is also called machine Mach number to emphasize its importance. In calculation of tip speed, head coefficient is needed that should be extract from Fig. 2 .

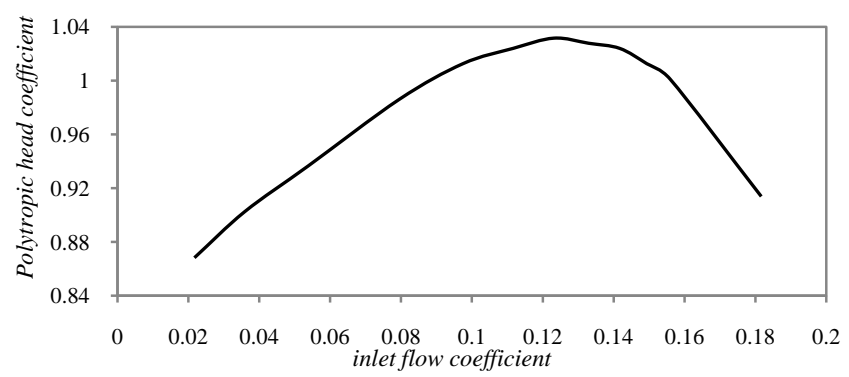

Fig. 2. Head coefficient verses flow coefficient [1].

\section{IMPELLER INLET SIZING}

As Fig. 2, in impeller inlet optimum eye diameter, blade inlet angle and blade inlet width should be calculated. Therefore, suction volume flow must be corrected for eye section by the following correction factors:

- Heat-up expansion

- Inlet loss expansion

- Inlet expansion

- Recirculation

- Cover-Disk leakage

To calculate heat-up expansion and cover-disk leakage, it is necessary to obtain balance piston mass flow and cover-disk mass flow (see Appendix).

Applying these coefficients, actual static suction volume flow at eye area will be achieved. This parameter is necessary due to obtain optimum eye diameter by implementing the condition mentioned below.

$$
\frac{d\left(\left(w_{1} / u_{2}\right)^{2}\right)}{d\left(d_{E} / d_{2}\right)}=0
$$

Clearly, by rearranging relative speed correlation as a function of eye diameter and taking the derivative with respect to eye diameter, it is concluded as:

$$
\left(\frac{d_{E}}{d_{2}}\right)_{o p t}=\sqrt{\left(\frac{d_{H}}{d_{2}}\right)^{2}+\left(2\left(\frac{K \emptyset}{d_{1} / d_{E}}\right)^{2}\right)^{1 / 3}}
$$

where $d_{1} / d_{E}$ is approximately $0.83-0.88$ for impellers with 3D-blades and approximately 1.0-1.12 for impellers with 2D-blades. In addition, to reduce resultant stresses at impeller eye, it is usual to make actual eye diameter some $5 \%$ smaller than the obtained optimum value. This reduction is in price of $1 \%$ relative velocity increment, which consequently maximizes impeller inlet losses [1].

To calculate blade inlet angle, inlet velocity triangle is used. The eq. 6 is derived from continuity equation to result impeller inlet width.

$$
b_{1}=\frac{\dot{V_{0 t}} v_{T} v_{I L} v_{I E} \frac{f}{\eta_{L}}}{\left(\frac{\pi}{4}\right) d_{2}^{2} u_{2} 4 \frac{d_{1}}{d_{E}} \frac{d_{E}}{d_{2}} \frac{1}{d_{2}} \frac{c_{m 1}}{u_{2}}}
$$

\section{IMPELLER EXIT SIZING}

In this section, one of the major discussions is around calculation of impeller exit width. Basis for calculation of exit width (eq. 7), is continuity equation, however, it is involved in obtaining exit flow coefficient and impeller volume ratio. Besides, blade exit angle is not a point to debate due to its consistency for inlet flow coefficient greater than 0.06 [1]. Furthermore, slip factor, work input factor and definition of reaction are presented in this section as well.

$$
\frac{b_{2}}{d_{2}}=\varphi \frac{\dot{V}_{3} / \dot{V}_{0 t}}{4 \varphi_{3}} \frac{f}{\eta_{L}}
$$

It has been shown from well proven experience that the impeller exit flow coefficient for the subjected impeller has to be function of the flow coefficient (Fig. 3).

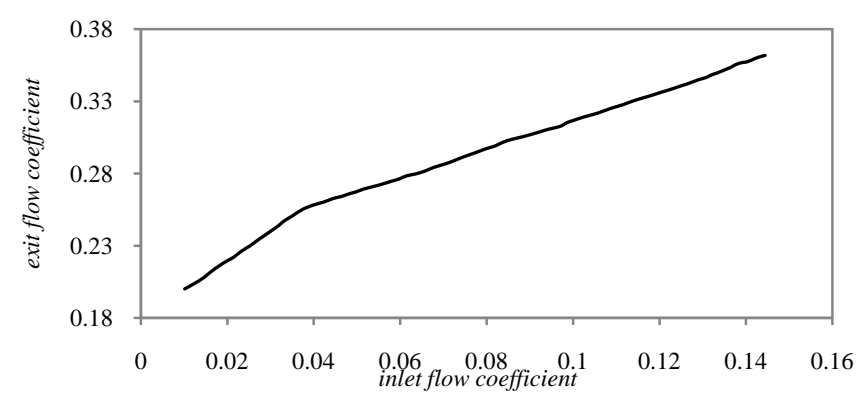

Fig. 3. Exit flow coefficient as function of inlet flow coefficient [1].

Impeller slip factor, which is the dimensionless thermodynamic work, can be calculated from the following empirical correlation [5]:

$$
s^{*}=1-\frac{\sqrt{\sin \beta_{2 B}}}{z^{0.7}}-\frac{\varphi_{3}}{\tan \beta_{2 B}}+\Delta s^{*}
$$

where $\Delta s^{*}$ is an experimentally determined correction. By adding disk friction and shroud leakage work to slip factor, work input factor is resulted. The equation mentioned above plus some experimental and theoretical correction can be used for work input factor. Therefore, as a side effect, a different correction $(\Delta s=-0.017 \pm 0.005)$ is employed.

Reaction is defined as a ratio of static enthalpy to total enthalpy. The correlation can be rearranged in form of exit flow coefficient and work input factor as follow:

$$
r=1-\frac{\varphi_{3}^{2}+s^{2}}{2 s}
$$

\section{DIFFUSER AERODYNAMIC}

It is crucial to calculate the change of state in the annular diffuser so as to result in inlet condition at the return vane 
channel and the volute. The most notable issue is the friction of flow along the diffuser walls, which makes change in angular momentum. Calculation of change of angular momentum undertook through the following equation:

$$
\dot{m} d\left(r c_{u}\right)=M_{f}
$$

Adding frictional term and rearranging the formula published in [3], the angular momentum of viscous flow at an arbitrary location of diffuser can be calculated as per Eq. 11 .

$$
\begin{gathered}
r c_{u}=r_{2} c_{u 3}-F \\
=r_{2} c_{u 3}-\frac{1}{b_{2} r_{2} c_{m 3} \rho_{3}} \int_{r_{2}}^{r} c_{f} \rho c_{u} \sqrt{c_{u}^{2}+c_{m}^{2}} r^{2} d r
\end{gathered}
$$

The procedure presented in [3], recommends to following a stepwise method, in which calculation carried out for the dimensionless form of Eq. 11.

\section{VOLUTE AND EXIT DiffuSER SIZING}

The aerodynamic design of the volute o scroll remains a rather qualitative and somewhat subjective process [4]. The quadratic internal volute design procedure performs through calculating any through-flow area at entire volute azimuth as per the continuity equation. Rearranging this equation for volute section, Eq. 12 is obtained.

$$
A=\frac{\dot{V}_{\vartheta}}{c_{u}}=\frac{\vartheta}{360} \frac{\dot{V}_{V}}{c_{u 4 i d} \frac{c_{u 4}}{c_{u 4 i d}} \frac{r_{4}}{r}}
$$

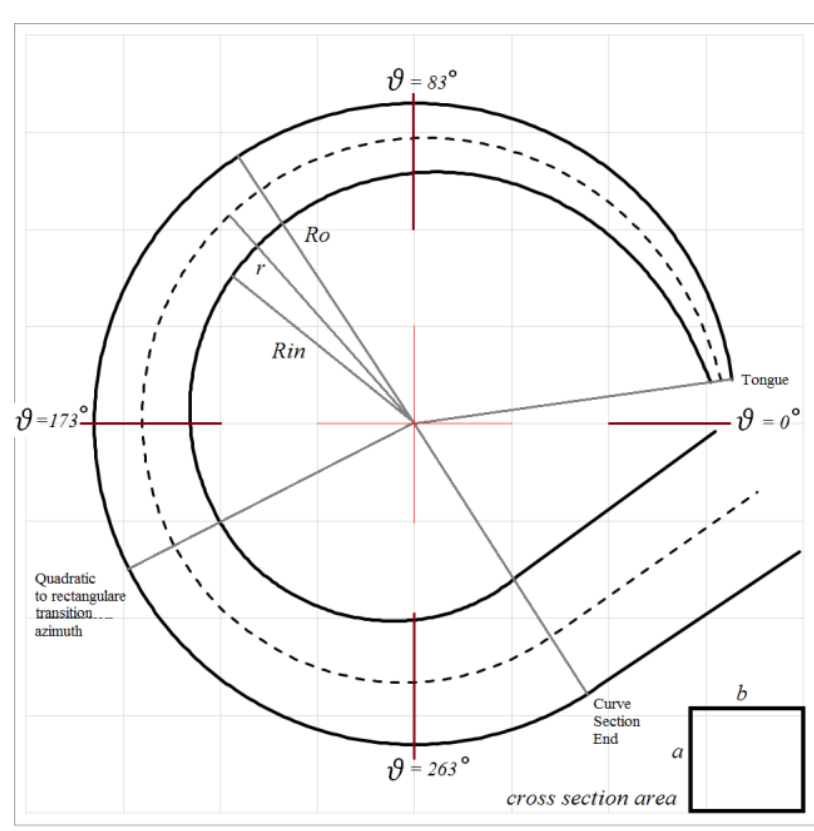

Fig. 4. Axial view of volute:

Ro outer radius, Rin inner radius, $r$ center of gravity radius.

Volute consists of two main section, namely curved and straight sections. The curved section start with a quadratic cross section area, which is became rectangular at a specified wrap angle. From the beginning of rectangular section $b$ (radial cross section width) is considered constant and $a$ (axial cross section width) increments.
Moreover, the straight section simply is an area growth in order to convert more kinetic energy into static pressure. Minimum area incensement is calculated from [1]:

$$
\frac{A_{6}}{A_{5}} \geq \frac{360}{\vartheta_{5}}
$$

where index 5 and 6 are at inlet and exit of straight section, respectively.

In order to take in account volute area in mentioned calculation, Eq. 12 is rearranged as follow:

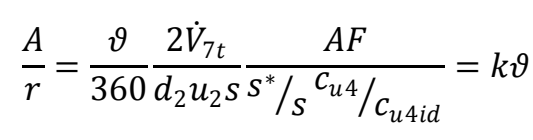

The area factor $(A F)$ normally is 1.0, however it can assume smaller, i.e. 0.7-1.0, which will shift the entire stage characteristic curve to smaller volume flows with a higher head rise to surge [1].

As Fig. 5 presents, by reducing volute effective area the maximum efficiency drops by less than $1 \%$ and as $\varphi$ increases the difference between full volute area curve and reduced volute area curve become more notable [1].

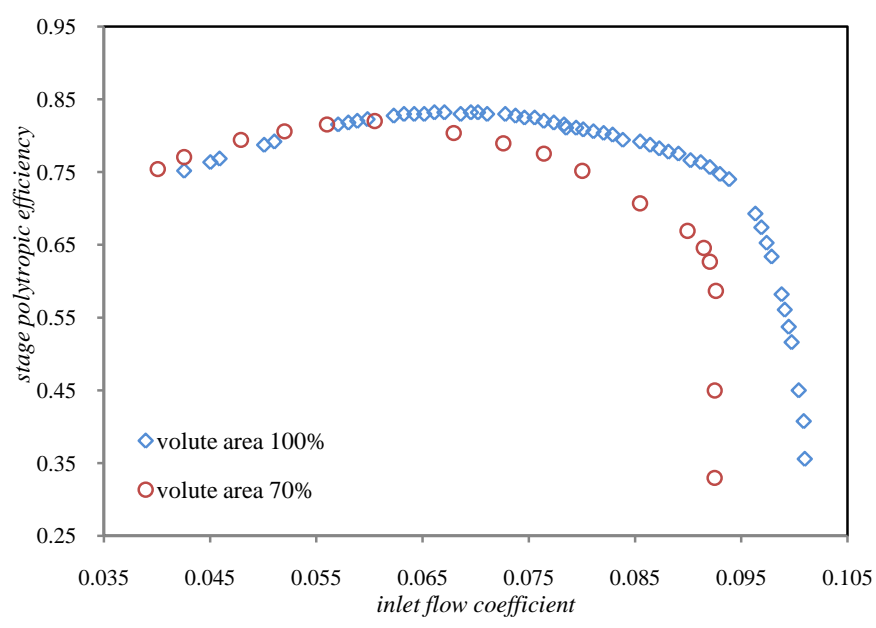

Fig. 5. Influence of the volute size on compressor pol. Efficiency. [1]

Each volute is followed by a conical exit diffuser. Since most of the exit diffusers in compressors have a rectangular inlet areas and circular exit area, the stall tends to occur at smaller area ratios [1]. Pressure recovery factor, as per eq. 15, is mostly affected by exit diffuser length and area ratio.

$$
c_{p}=\frac{p_{2 s t a t}-p_{1 s t a t}}{p_{1 \text { tot }}-p_{1 s t a t}}
$$

where "stat" refers to static and "tot" refers total. According to [1] acceptable value for pressure recovery is more than 0.5 , which means area ratio more than 0.3 as well as length/diameter ratio more than 4.5. These values are preferable due to exit diffuser design is secured not to go on stall.

\section{RESULTS}

In this section the results obtained by the adapted design procedure with available data comparison is presented. 
TABLE I: GAS PSEUDO-CRITICAL PROPERTIES

\begin{tabular}{|l|c|c|c|c|c|}
\hline \hline \multirow{2}{*}{ Parameter } & \multirow{2}{*}{ Unit } & \multicolumn{2}{|c|}{ Design } & \multicolumn{2}{c|}{ Ref. Data, [1] } \\
\cline { 3 - 6 } & & St. 1 & St. 2 & St. 1 & St. 2 \\
\hline Suc. comp. fac. & - & 0.8881 & 0.9006 & 0.883 & 0.889 \\
\hline Disc. comp. fac. & - & 0.9006 & 0.9157 & 0.889 & 0.897 \\
\hline Suc. isen. vol. exp. & - & 1.3769 & 1.3764 & 1.339 & 1.358 \\
\hline Disc. isen. vol. exp. & - & 1.3764 & 1.3844 & 1.358 & 1.381 \\
\hline Suc. isen. temp. exp. & - & 1.3211 & 1.3077 & 1.319 & 1.312 \\
\hline Disc. isen. temp. exp. & - & 1.3077 & 1.2944 & 1.312 & 1.303 \\
\hline \hline
\end{tabular}

TABLE II: IMPELLER INLET \& EXIT

\begin{tabular}{||l|c|c|c|c|c||}
\hline \multirow{2}{*}{ Parameter } & \multirow{2}{*}{ Unit } & \multicolumn{2}{|c|}{ Design } & \multicolumn{2}{c||}{ Ref. Data, [1] } \\
\cline { 3 - 6 } & & St. 1 & St. 2 & St. 1 & St. 2 \\
\hline Impeller diameter & $\mathrm{mm}$ & 304 & 302 & 304 & 302 \\
\hline Tip speed & $\mathrm{m} / \mathrm{s}$ & 222.39 & 220.9 & 222.39 & 220.9 \\
\hline Inlet flow coeff.(ref d2) & - & 0.104 & 0.0938 & 0.1024 & 0.0907 \\
\hline Static/Process vol. flow & - & 1.0356 & 1.0315 & 1.044 & 1.037 \\
\hline Opt. eye dia. ratio & - & 0.6423 & 0.6481 & 0.665 & 0.655 \\
\hline Rel. imp. in. width & - & 0.1419 & 0.1296 & 0.139 & 0.124 \\
\hline Rel. imp. ex. Width & - & 0.0758 & 0.0713 & 0.0761 & 0.0688 \\
\hline Exit flow coeff. & - & 0.3208 & 0.3098 & 0.314 & 0.310 \\
\hline Work. in. factor & - & 0.5981 & 0.6073 & 0.604 & 0.607 \\
\hline Reaction & - & 0.6149 & 0.6173 & 0.616 & 0.617 \\
\hline
\end{tabular}

TABLE III: VOLUTE AND EXIT DIFFUSER

\begin{tabular}{|l|c|c|c|}
\hline \hline \multirow{2}{*}{ Parameter } & \multirow{2}{*}{ Unit } & Design & Ref. Data, [1] \\
\cline { 3 - 4 } & & St. 2 & St. 2 \\
\hline Exit volume flow & $\mathrm{m}^{3} / \mathrm{s}$ & 1.3223 & 1.258 \\
\hline Slip/Work in. fac. ratio & - & 0.9785 & 0.985 \\
\hline Max. axial width & $\mathrm{mm}$ & 108.4 & 105.9 \\
\hline Inlet dimension of ex. dif. & $\mathrm{mm}^{2}$ & 18740 & 19909.2 \\
\hline Area ratio & - & 4.028 & 3.55 \\
\hline Exit dif. Length & $\mathrm{mm}$ & 70.8475 & 81.8 \\
\hline Pressure recovery coeff. & - & 0.50571 & 0.51 \\
\hline \hline
\end{tabular}

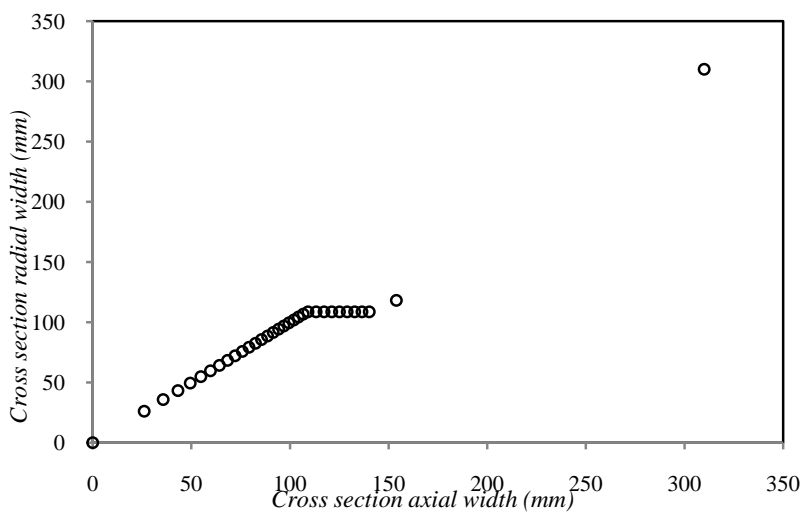

Fig. 6. Volute cross section area

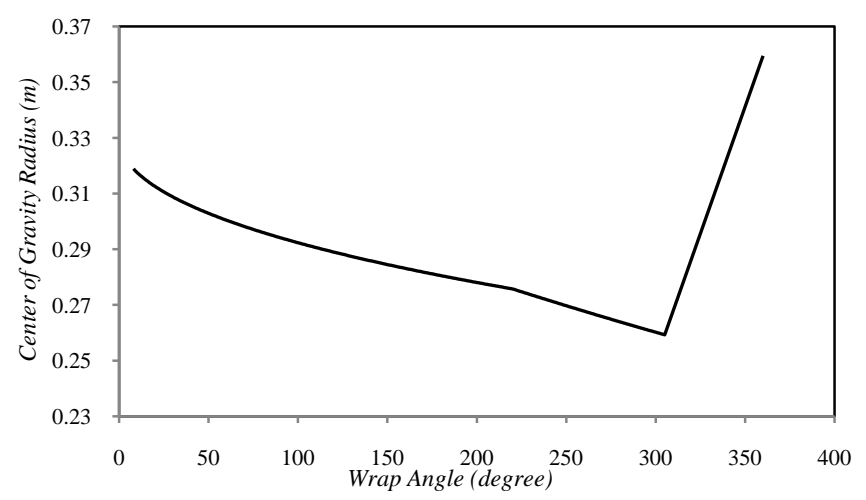

Fig. 7. Center of gravity radius in different volute azimuths

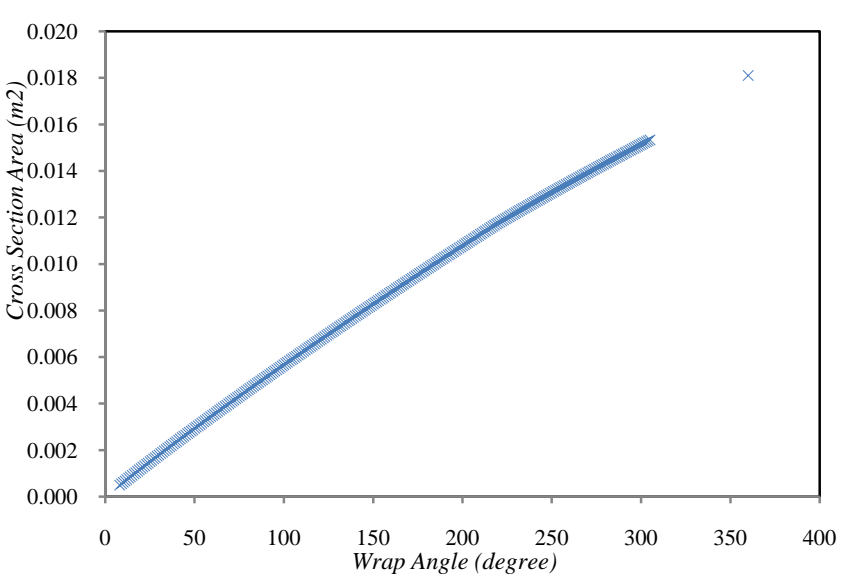

Fig. 8. Cross section area in different volute azimuths

\section{CONCLUSION}

In this paper, an adapted procedure to design different sections of centrifugal compressor was presented. Reviewing the procedure reveals that, there are essential parameters such as tip speed Mach number (machine Mach number), inlet flow coefficient, impeller diameter and so forth, which play major roles in determining compressor polytropic head, and consequently, compressor polytropic efficiency. Therefore, following the presented method concludes in results, which its deviation from reference data is considerably low.

There are some differences between designed volute with referred one. This dissimilarity rises on the grounds that the mentioned method considers minimum optimum values rather than most efficient ones, which lead to an economical design.

\section{APPENDIX}

The inevitable parasitic flows through the labyrinth seals of cover-disk and balance piston can be satisfactorily calculated with the following numerical formula [5]:

$$
\Delta \dot{m}=\frac{\xi \varepsilon A p_{1}}{\sqrt{10^{5} Z_{1} R\left(273.15+t_{1}\right)}}
$$

where

$\xi \approx 0.7$ : coefficient of flow for interlocking seals

$\xi \approx 0.7$ : coefficient of flow for see-through seals

$\varepsilon=\mathrm{f}\left(\mathrm{n}, \mathrm{p}_{2} / \mathrm{p}_{1}\right)$ : seal factor

$A=D \pi s:$ gap area $\left(\mathrm{mm}^{2}\right)$

$D$ : seal diameter $(\mathrm{mm})$

$\mathrm{s}$ : clearance $(\mathrm{mm})$

$\mathrm{p}_{1} / \mathrm{p}_{2}$ : pressure upstream/downstream of seal (bar)

$\mathrm{Z}_{1}$ : compressibility factor upstream of seal

$\mathrm{R}$ : gas constant $(\mathrm{kJ} / \mathrm{kg} \mathrm{K})$

$\mathrm{t}_{1}$ : temperature upstream of seal $\left({ }^{\circ} \mathrm{C}\right)$

$\mathrm{n}$ : number of sealing tips

Number of sealing is normally 5 for cover -disk interlocking seals and 20 to 30 for balance-piston interlocking seals [1]. Since pressure ratio and number of sealing tips are known, using fig. 9, seal factor can be achieved. 


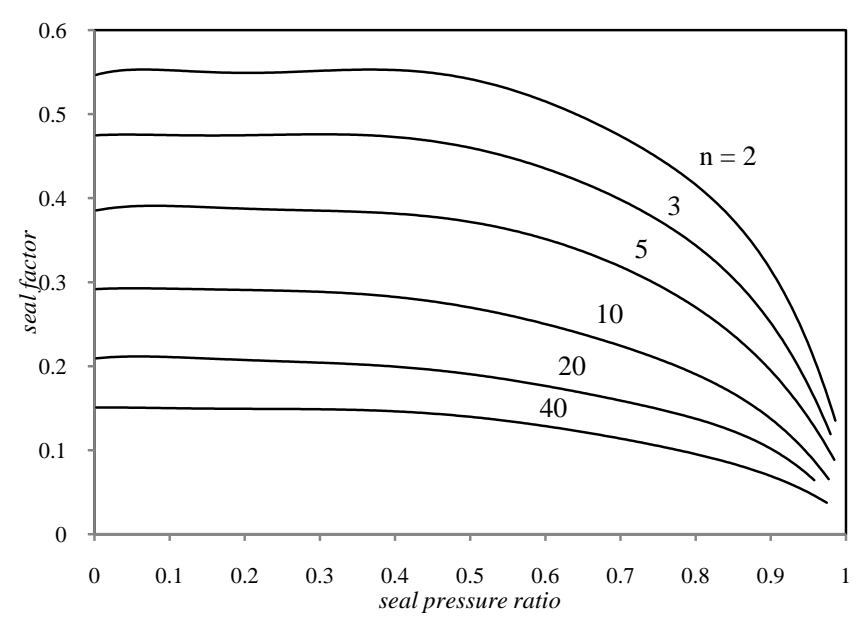

Fig. 9. Labyrinth seal factor: $n$ number of sealing tips, [1]

\section{REFERENCES}

[1] H. K. Lüdtke, Process Centrifugal Compressor: Basics, Function, Operation, Design, Application, Springer, $1^{\text {st }}$ Edition, 2004, ch. 6.

[2] R. C. Reid, J. M. Prausnitz, and B. Poling, The Properties of Gases \& Liquids, McGraw Hill, 1987, ch. 4, pp. 74-87.

[3] W. Traupel, Die Theorie der Strömung durch Raialmachinen, G. Braun, Karlsruhe, Germany (in German)

[4] R. H. Aungier, Centrifugal Compressors: A Strategy for Aerodynamic Design and Analysis, ASME Press, New York, USA, 2000, ch. 11, pp. 195-200.

[5] BORSIG Pocket Book, Borsig GmbH Berlin, $7^{\text {th }}$ ed., 1994.

[6] F. J. Wiesner, "A Review of Slip Factors for Centrifugal Impellers," ASME Paper 66-WA/FE, 1966.

[7] N. A. Caumpsty, Compressor Aerodynamics, Longman, 1989, ch. 1, pp. $1-11$, ch. 6 , pp. $220-236$
[8] K. H. Lüdtke, "Twenty years of experience with a modular design system for centrifugal compressors," Presented at $21^{\text {th }}$ Turbomachinery Symposium, Houston, TX, USA, 1992.

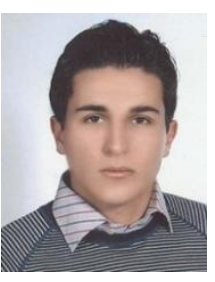

Hassan Ali Marefat is a B.Sc. candidate in Mechanical Engineering at Islamic Azad University, Science \& Research Campus. He is already working in Turbomachinery field, as a researcher in Design Department of Oil Turbo Compressor Co.

Majid Reza Shahhosseini is a Ph.D. graduated from Sharif University of Technology. He is working in Oil TurboCompressor Equipment Company (OTCE) as design department manager in field of turbo-compressor.

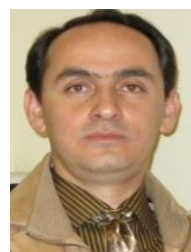

Mohammad Ali Ashjari is a graduated Ph.D. in thermo-fluid. His major field is enhanced oil recovery (EOR) and reservoir simulation. Gas injection projects using macro centrifugal compressors are his most interested fields.

He is now a faculty member of Mechanical Engineering Dept. in Islamic Azad university Jolfa branch. He actively works on design and reverse engineering of centrifugal compressors. His recent publication is Application of Dry Gas Seal - Advantages and Limitations (in Persian). 